International Journal of Advanced Astronomy, 8(2) (2020) 35-36
International Journal of Advanced Astronomy
SPC
Website $:$ www.sciencepubco.com/index.php/IJAA
Research paper

\title{
The Dazzling Comet C/2020 F3 (NEOWISE): The Comet of The Century
}

\author{
M. Khalil ${ }^{1}$ *, M. Said ${ }^{1}$, H. Osman ${ }^{1}$, N. Younis ${ }^{2}$, N. Khaled ${ }^{1}$, Y. Amr ${ }^{1}$, N.A. Mohamed ${ }^{1}$, A. Ibrahim ${ }^{1}$ \\ ${ }^{1}$ Faculty of Engineering, October University for Modern Sciences and Arts (MSA), Giza, Egypt \\ ${ }^{2}$ Department of English and American studies, University of Vienna, Austria \\ *Corresponding authorE-mail: mkibrahim@msa.eun.eg
}

\begin{abstract}
The newfound long period comet NEOWISE has the potential to be one of the brightest comets ever seen. The comet which was visible to the naked eye is considered as the comet of the century. This icy object was discovered on March 27, 2020. In this paper, we dive into some fascinating facts about NEOWISE comet that make such comet unique.
\end{abstract}

Keywords: NEOWISE (C/2020 F3) Comet; Bright Comets; Comet of the Century.

\section{Introduction}

In 2020, the Earth was on a date with an icy visitor from the deep space. This cosmic visitor was the comet NEOWISE. The long period comet NEOWISE is one of the brightest comets ever. It was discovered on March 27, 2020 by NASA's Wide-field Infrared Survey Explorer (WISE) space telescope and then classified as a comet on March 31, 2020. It is formally known as comet C/2020 F3. It is the first visible comet to the naked eye after the comet Hale Bopp which was discovered in 1995. The rest of the paper is organized as follows. In Section 2, some features of NEOWISE comet are presented while in section 3 we discuss the comet's composition and origin. Our conclusion and recommendations are presented in section 4

\section{Some features of NEOWISE comet}

Recently, comet NEOWISE has become in the top news headlines. The nucleus of NEOWISE comet is about $5 \mathrm{~km}$ wide and it is travelling with a speed of $231,000 \mathrm{~km} / \mathrm{h}(64 \mathrm{~km} / \mathrm{s})$. NEOWISE has two tails like any other comet as shown in figure 1 . One of them is a dust tail while the other is an ion tail. But satellite data indicates that NEOWISE comet has a dust tail and possibly two ion tails. The length of its dust tail is about 16 million $\mathrm{km}$. The elliptical orbit of this comet is very long. It is about 6,800 years before it returns back. In other words, people who will see it would have lived during the fifth millennium BC. On March 27, 2020 the object was discovered by a team using WISE telescope under NEOWISE program, and then classified as a comet on March 31, 2020 and named NEOWISE in April. NEOWISE made its closest approach to Earth on July 22, 2020 with a distance of 103 million km.

\section{The comet's composition and origin}

The comet NEOWISE is considered as one of the brightest comets because it got much closer to the sun than most other comets. The brightness of the comet enables scientists to get a lot of data than any other comet. There is a lot of water in NEOWISE (about 13 million Olympic swimming pools of water). The comet's head is green due to the existence of diatomic carbon $\mathrm{C} 2$ while the tail of the comet is blue because of the abundance of carbon monoxide (CO). Comet NEOWISE originates in our solar system (only two objects have been discovered in the interstellar space: Oumuamua and Comet Borisov). Monte Carlo computer models have been used in order to simulate the sodium tail and to estimate outgassing rates and speeds.

\section{Conclusion and recommendations}

In this work, an overview of the comet NEOWISE is presented to highlight the importance of studying this comet which is considered as the comet of the century. One of the main aims of this paper is to motivate research committees around the world to study the gathered data of this unique comet. NEOWISE proves how important it is for us to develop our detection systems against such threats which could strike Earth without warning. 


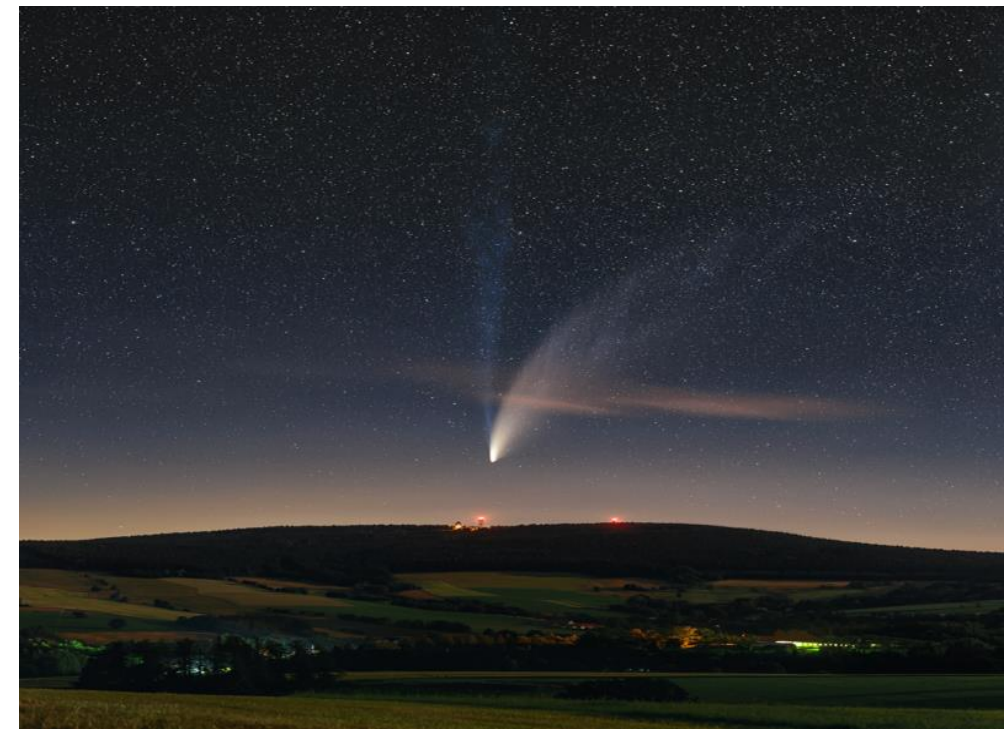

Fig. 1: Long NEOWISE's Ion Tail.

Credits: Long NEOWISE’s ion tail, Petr Horálek Photography, (C) 2020 by Petr Horálek, used with permission.

\section{Acknowledgement}

We are deeply grateful to Mr. Petr Horálek for giving us a permission to use his valuable picture of NEOWISE comet.

\section{References}

[1] Hadamcik, E., Levasseur-Regourd, A.C., Hines, D.C., Sen, A.K., Lasue, J. and Renard, J.B., 2016. Properties of dust particles in comets from photometric and polarimetric observations of 67P. Monthly Notices of the Royal Astronomical Society,462(Suppl_1), pp. S507-S515. https://doi.org/10.1093/mnras/stx030.

[2] Khalil, M., Said, M., Osman, H., Ahmed, B., Ahmed, D., Younis, N., Maher, B., Osama, M. and Ashmawy, M., Big data in astronomy: from evolution to revolution, International Journal of Advanced Astronomy, 7 (1) (2019) 11-14. https://doi.org/10.14419/ijaa.v7i1.18029.

[3] Khalil, M., Said, M., Osman, H., Younis, N., Khaled, N., Amr, Y., Mohamed, N.A., Ibrahim, A., Dark comets: the cosmic catastrophic threat to earth, International Journal of Advanced Astronomy, v. 8, n. 1, p. , 8 (1) (2020) 27-28. https://doi.org/10.14419/ijaa.v8i1.30740.

[4] Space.com: Comet NEOWISE: 10 big questions (and answers) about the icy wanderer.

[5] https://www.space.com/comet-neowise-strange-facts.html.

[6] Science the Wire: With Neowise in Our Sights, Earthlings Go Comet-Spotting.

[7] https://science.thewire.in/the-sciences/comet-neowise-solar-system-time-capsuleneo/\#: : :text=True $\% 2 \mathrm{C} \% 20$ Earth\%20is\%20much\%20more,against $\% 20$ such $\% 20$ threats\%20from\%20space.

[8] NASA: (2020, July 15) Comet NEOWISE Seen in an Aurora-Filled Sky.

[9] https://www.nasa.gov/image-feature/goddard/2020/comet-neowise-seen-in-an-aurora-filled-sky.

[10] NASA: (2020, July 8) Comet NEOWISE Sizzles as It Slides by the Sun, Providing a Treat for Observers.

[11] https://www.nasa.gov/feature/jpl/comet-neowise-sizzles-as-it-slides-by-the-sun-providing-a-treat-for-observers.

[12] NASA: (2020, July 10) NASA's Parker Solar Probe Spies Newly Discovered Comet NEOWISE.

[13] https://www.nasa.gov/feature/goddard/2020/nasa-s-parker-solar-probe-spies-newly-discovered-comet-neowise/. 